\title{
Axisymmetric Scholte Waves and Special Features of Propagation
}

\author{
Chunlei Bian ${ }^{a}$, Ji $\mathrm{WANG}^{a, b, *}, \mathrm{Bin} \mathrm{HuAnG}^{a, b}$, \\ LOngtao XiE ${ }^{a, b}$, LiJun Yi ${ }^{a, b}$, Lili YUAN ${ }^{a, c}$, \\ HONGLANG $\mathrm{LI}^{d}$ AND YAHUI TIAN ${ }^{e}$ \\ ${ }^{a}$ Piezoelectric Device Laboratory, School of Mechanical Engineering and Mechanics, \\ Ningbo University, 818 Fenghua Road, Ningbo, 315211 Zhejiang, China \\ ${ }^{b}$ TXC-NBU Joint Center of Research, School of Mechanical Engineering and Mechanics, \\ Ningbo University, 818 Fenghua Road, Ningbo, 315211 Zhejiang, China \\ ${ }^{c}$ School of Civil and Environmental Engineering, Ningbo University, \\ 818 Fenghua Road, Ningbo, 315211 Zhejiang, China \\ ${ }^{d}$ National Center for Nanoscience and Technology, \\ 11 Beiyitiao Road, Zhongguancun, 100190 Beijing, China \\ ${ }^{e}$ Institute of Acoustics, Chinese Academy of Sciences, \\ 21 West Beisihuan Road, Zhongguancun, 100190 Beijing, China
}

Received: 30.03.2021 \& Accepted: 14.04.2021

Doi: $10.12693 /$ APhysPolA.139.710

*e-mail: wangji@nbu.edu.cn

\begin{abstract}
As a special wave mode propagating in the interface between an infinite elastic solid and fluid, the Scholte waves are well known for their existence and frequency with distinct properties. The analysis and features are usually presented through the formulation in Cartesian coordinates, while the essential features of the phase velocity and wave patterns are also similar in other coordinates on the basis of equivalence. A variation of the Scholte wave features with a coordinate framework should be examined for possible insights related to mathematical solutions and applications besides the known properties. Using a systematic formulation with cylindrical coordinates and subsequent solutions in the Bessel functions, it is proved that the Scholte waves will attenuate with the increase of a radius in an axisymmetric case, which is different from the results in the Cartesian coordinate system. In addition, the particle trajectory will also vary due to the changes of the waveform. The examination of such features in a systematic analysis should play a prominent role in engineering applications of wave propagation associated with cylindrical solids.
\end{abstract}

topics: Scholte wave, axisymmetric, propagation, velocity

\section{Introduction}

In elastic solids, there are many different waves propagating with special features and conditions like the Rayleigh waves and Stoneley waves, to name just a few of the popular wave types. Examining wave propagation in elastic solids, a few typical wave modes with distinct characteristics and solutions have been popularly known as part of the theory and applications of wave propagation in different configurations of structures and materials. Among the few widely known wave modes, the Scholte waves are well examined in the literature for their unique features like being nondispersive with material properties. If the Stoneley waves propagate between the interface of an infinite elastic solid and fluid, they are conventionally called the Scholte waves $[1,2]$. Cagniard presented a general condition for which the Scholte waves exist, which requires no special condition of material properties [3]. Complete solutions and the examination of properties of the Scholte waves are available in many textbooks and monographs, and they are also the topic covered in the fundamental theory of wave propagation. Moreover, almost all earlier studies on these popular wave types are done with Cartesian coordinates, leaving later students and researchers wondering whether there would be any special features if other coordinates, say the axisymmetric ones, were used.

As far as the wave patterns and properties and structures with basic configurations different from rectangular shapes are concerned, it is important to find the wave propagation with an appropriate choice of coordinate systems, which have also been presented in many popular textbooks and monographs by Ewing [4], Achenbach [5], Graff [6], Eringen [7], Rose [8], and some dissertations [9-12] with 
details on specific wave modes. Strangely enough, details of axisymmetric waves are not given in major literature including the above-mentioned references for the essential modes. Of course, it does not mean that there are difficulties in obtaining the solutions and making necessary comparisons with known results in rectangular coordinates. It just shows a general conviction that the solutions and essential features are exactly the same and independent from the coordinates. This, of course, is correct to some extent but there are specific features with coordinate system dependence and it can be easily hidden if not examined in detail, as shown in our recent papers $[13,14]$. In recent studies of the Rayleigh waves and Stoneley waves in axisymmetric solids, it is found that the deformation, or the trajectory of particles, is quite different from solutions in the Cartesian coordinate system, although the velocities are the same and the wave patterns are the same for a larger radius. Since the solutions are in the Bessel functions, there are some special features to be better understood through comparison with a different coordinate framework. It is believed that a close examination of the Scholte waves with axisymmetric coordinates will also reveal some special properties from the axisymmetric solutions.

\section{Governing equations and solutions of axisymmetric Scholte waves}

The Scholte waves exist in a liquid-solid interface with a semi-infinite solid in contact with fluid $[15,16]$. To analyze the wave modes, one begins with the assumption that the axisymmetric Scholte waves propagate along the radius $r$ and decay along the depth $z$ with cylindrical coordinates in the liquid-solid interface along the plane $z=0$. This system, schematically shown in Fig. 1, is characterized by the density and Lamé constants $\bar{\rho}$, $\bar{\lambda}$ for the liquid (Material 1) and $\rho, \lambda$ for the solid (Material 2), respectively. Material 2 is described, in addition, by the parameter $\mu$ defined as shear modulus.

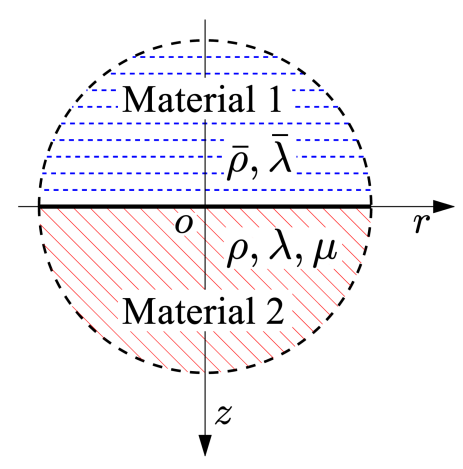

Fig. 1. The interface of a semi-infinite liquid and solid material in cylindrical coordinates.
To retain the axisymmetric property in this problem, the angular coordinate $\theta$ and the circumferential displacement component $u_{\theta}$ must vanish. Hence, the displacement vector is simplified to

$$
\boldsymbol{u}(r, z, t)=u_{r}(r, z, t) \boldsymbol{e}_{r}+u_{z}(r, z, t) \boldsymbol{e}_{z},
$$

where the displacements $u_{r}$ and $u_{z}$ are the functions of the $r$ and $z$ coordinates, while $t$ is the time.

Through the Helmholtz decomposition, a scalar potential $\phi$ and a vector potential $\boldsymbol{H}=H_{r} \boldsymbol{e}_{r}+$ $H_{\theta} \boldsymbol{e}_{\theta}+H_{z} \boldsymbol{e}_{z}$ should be introduced to simplify the problem further for possible solutions [5]. With (1), it only needs to keep the component $H_{\theta}$ in $\boldsymbol{H}$, and the displacements can be decomposed into

$\boldsymbol{u}(r, z, t)=\nabla \phi(r, z, t)+\nabla \times\left(H_{\theta}(r, z, t) \boldsymbol{e}_{\theta}\right)$, where

$$
\begin{aligned}
& \nabla \phi=\frac{\partial \phi}{\partial r} \boldsymbol{e}_{r}+\frac{\partial \phi}{\partial z} \boldsymbol{e}_{z} \\
& \nabla \times\left(H_{\theta} \boldsymbol{e}_{\theta}\right)=-\frac{\partial H_{\theta}}{\partial z} \boldsymbol{e}_{r}+\frac{1}{r} \frac{\partial\left(r H_{\theta}\right)}{\partial r} \boldsymbol{e}_{z} .
\end{aligned}
$$

Clearly, the displacement components in potentials are

$$
\begin{gathered}
\boldsymbol{u}(r, z, t)=\left(\frac{\partial \phi}{\partial r}-\frac{\partial H_{\theta}}{\partial z}\right) \boldsymbol{e}_{r} \\
+\left(\frac{\partial \phi}{\partial z}+\frac{1}{r} \frac{\partial\left(r H_{\theta}\right)}{\partial r}\right) \boldsymbol{e}_{z}
\end{gathered}
$$

By substituting the displacement vector $u$ into the Lamé equations of motion in elasticity [17, 18]:

$$
(\lambda+\mu) \nabla \cdot(\nabla \boldsymbol{u})+\mu \nabla^{2} \boldsymbol{u}=\rho \ddot{\boldsymbol{u}},
$$

the problem should be reduced further with a simplification of the two governing equations for $\phi$ and $H_{\theta}$ to

$$
\begin{aligned}
& \nabla^{2} \phi=\frac{1}{c_{\mathrm{L}}^{2}} \ddot{\phi}, \\
& \nabla^{2} H_{\theta}-\frac{1}{r^{2}} H_{\theta}=\frac{1}{c_{\mathrm{T}}^{2}} \ddot{H}_{\theta},
\end{aligned}
$$

where $c_{\mathrm{L}}=\sqrt{(\lambda+2 \mu) / \rho}$ and $c_{\mathrm{T}}=\sqrt{\mu / \rho}$ are the longitudinal and transverse wave velocities, respectively. In the cylindrical coordinate system

$$
\begin{aligned}
& \nabla^{2} \phi=\frac{1}{r} \frac{\partial}{\partial r}(r \phi)+\frac{\partial^{2} \phi}{\partial z^{2}}, \\
& \nabla^{2} H_{\theta}=\frac{1}{r} \frac{\partial}{\partial r}\left(r H_{\theta}\right)+\frac{\partial^{2} H_{\theta}}{\partial z^{2}} .
\end{aligned}
$$

With an introduction of a further transformation

$$
H_{\theta}=-\frac{\partial \psi(r, z, t)}{\partial r},
$$

the displacements are now

$$
\begin{gathered}
\boldsymbol{u}(r, z)=\left(\frac{\partial \phi}{\partial r}+\frac{\partial^{2} \psi}{\partial r^{2}}\right) \boldsymbol{e}_{r} \\
+\left(\frac{\partial \phi}{\partial z}+\frac{\partial^{2} \psi}{\partial z^{2}}-\frac{1}{c_{\mathrm{T}}^{2}} \ddot{\psi}\right) \boldsymbol{e}_{z}
\end{gathered}
$$

and (8) is equivalent to

$$
\nabla^{2} \psi=\frac{\ddot{\psi}}{c_{\mathrm{T}}^{2}}+g(z),
$$

in which $g(z)$ is an arbitrary function of $z$. Let $g(z)=0$, then one reaches $\nabla^{2} \psi=\frac{1}{c_{\mathrm{T}}^{2}} \ddot{\psi}$. 
Therefore, the equivalence of (7) and (8) is

$$
\begin{aligned}
& \nabla^{2} \phi=\frac{1}{c_{\mathrm{L}}^{2}} \ddot{\phi}, \\
& \nabla^{2} \psi=\frac{1}{c_{\mathrm{T}}^{2}} \ddot{\psi} .
\end{aligned}
$$

We are now dealing with standard wave propagation equations with wave velocities $c_{\mathrm{L}}$ and $c_{\mathrm{T}}$ of longitudinal and transverse waves. Exactly the same forms as in the case of the wave modes indicate that only a single equation has to be solved to obtain solutions to the problem. We will use the method of separation of variables through $\phi(r, z, t)=R(r) Z(z) \mathrm{e}^{\mathrm{i} \omega t}$, in which $\omega$ is the angular frequency of waves. The simplified form of (14) is written as

$$
\frac{\omega^{2}}{c_{\mathrm{L}}^{2}} R Z+\left(Z \frac{\mathrm{d}^{2} R}{\mathrm{~d} r^{2}}+\frac{Z}{r} \frac{\partial R}{\partial r}+R \frac{\mathrm{d}^{2} Z}{\mathrm{~d} z^{2}}\right)=0,
$$

with the wave number $k=\omega / c$ and the phase velocity $c$ of the Scholte waves.

Now the potential function $\phi$ is obtained as

$$
\phi(r, z, t)=\left(C \mathrm{e}^{\alpha z}+A \mathrm{e}^{-\alpha z}\right) J_{0}(k r) \mathrm{e}^{\mathrm{i} \omega t},
$$

where $A$ and $C$ are the undetermined constants, $\alpha$ is the decaying constant satisfying the relation $k^{2}=\alpha^{2}+\left(\omega / c_{\mathrm{L}}\right)^{2}$, and $J_{0}(k r)$ is the zeroth-order Bessel function of the first kind. The zeroth-order Bessel function of the second kind $Y_{0}(k r)$ is neglected because of the singularity at $r=0$.

Likewise, the second potential is

$$
\psi(r, z, t)=\left(B \mathrm{e}^{-\beta z}+D \mathrm{e}^{\beta z}\right) J_{0}(k r) \mathrm{e}^{\mathrm{i} \omega t},
$$

where $B$ and $D$ are also the undetermined constants and $\beta$ is the decaying constant from the relation $k^{2}=\beta^{2}+\left(\omega / c_{\mathrm{T}}\right)^{2}$.

As it is known, when $z>0$, the exponential function $\mathrm{e}^{\alpha z}$ is supposed to vanish because of unbounded solution with $z \rightarrow \infty$, and $\mathrm{e}^{\beta z}$, too. Thus, the potential function solutions are

$$
\begin{aligned}
& \phi=A \mathrm{e}^{-\alpha z} J_{0}(k r) \mathrm{e}^{\mathrm{i} \omega t}, \\
& \psi=B \mathrm{e}^{-\beta z} J_{0}(k r) \mathrm{e}^{\mathrm{i} \omega t} .
\end{aligned}
$$

With the substitution of (19) into the displacement function (12), the displacement field can be obtained as

$$
\begin{aligned}
& u_{r}=\frac{\partial \phi}{\partial r}+\frac{\partial^{2} \psi}{\partial r^{2}}= \\
& -k\left(A \mathrm{e}^{-\alpha z}-\beta B \mathrm{e}^{-\beta z}\right) J_{1}(k r) \mathrm{e}^{\mathrm{i} \omega t}, \\
& u_{z}=\frac{\partial \phi}{\partial z}+\frac{\partial^{2} \psi}{\partial z^{2}}-\frac{1}{c_{T}^{2}} \ddot{\psi}= \\
& -\left(\alpha A \mathrm{e}^{-\alpha z}-k^{2} B \mathrm{e}^{-\beta z}\right) J_{0}(k r) \mathrm{e}^{\mathrm{i} \omega t} .
\end{aligned}
$$

with $\alpha=k p, p=\sqrt{1-\left(c / c_{\mathrm{L}}\right)^{2}}, \beta=k q, q=$ $\sqrt{1-\left(c / c_{\mathrm{T}}\right)^{2}}$. Correspondingly, the stress fields are

$$
\begin{gathered}
\sigma_{z}=\lambda \nabla \cdot \boldsymbol{u}+2 \mu \frac{\partial u_{z}}{\partial z}= \\
\mu\left[\left(k^{2}+\beta^{2}\right) A \mathrm{e}^{-\alpha z}-2 k^{2} \beta B \mathrm{e}^{-\beta z}\right] J_{0}(k r) \mathrm{e}^{\mathrm{i} \omega t}
\end{gathered}
$$

$$
\begin{gathered}
\tau_{z r}=\mu\left(\frac{\partial u_{r}}{\partial z}+\frac{\partial u_{z}}{\partial r}\right)= \\
k \mu\left[2 \alpha A \mathrm{e}^{-\alpha z}-\left(k^{2}+\beta^{2}\right) B \mathrm{e}^{-\beta z}\right] J_{1}(k r) \mathrm{e}^{\mathrm{i} \omega t} .
\end{gathered}
$$

With $z<0$, the same procedure can be easily adapted to obtain - noting that the potential function $\bar{\psi}$ in displacement field must disappear because of no shear displacement in the liquid and similar materials like sand - the potential function $\bar{\phi}$ as

$$
\bar{\phi}=\bar{A} \mathrm{e}^{\bar{\alpha} z} J_{0}(k r) \mathrm{e}^{\mathrm{i} \omega t}
$$

with $\bar{\alpha}=k \bar{p}, \bar{p}=\sqrt{1-\left(c / \bar{c}_{L}\right)^{2}}$. The displacement field is

$$
\begin{aligned}
& \bar{u}_{r}=-k \bar{A} \mathrm{e}^{\bar{\alpha} z} J_{1}(k r) \mathrm{e}^{\mathrm{i} \omega t}, \\
& \bar{u}_{z}=\bar{\alpha} \bar{A} \mathrm{e}^{\bar{\alpha} z} J_{0}(k r) \mathrm{e}^{\mathrm{i} \omega t} .
\end{aligned}
$$

Then the stress field is

$$
\bar{\sigma}_{z}=-\bar{\lambda}\left(k^{2}-\bar{\alpha}^{2}\right) \bar{A} \mathrm{e}^{\bar{\alpha} z} J_{0}(k r) \mathrm{e}^{\mathrm{i} \omega t}
$$

Assuming the perfect interface of two half-spaces, boundary conditions are

$$
\begin{aligned}
& \left.\bar{u}_{z}\right|_{z=0}=\left.u_{z}\right|_{z=0}:-\alpha A+k^{2} B=\bar{\alpha} \bar{A} \\
& \left.\bar{\sigma}_{z}\right|_{z=0}=\left.\sigma_{z}\right|_{z=0}: \mu\left[\left(k^{2}+\beta^{2}\right) A-2 k^{2} \beta B\right]= \\
& -\bar{\lambda} \bar{A}\left(k^{2}-\bar{\alpha}^{2}\right) \\
& \left.\bar{\tau}_{r z}\right|_{z=0}=\left.\sigma_{z}\right|_{z=0}: 2 \alpha A-\left(k^{2}+\beta^{2}\right) B=0
\end{aligned}
$$

The above equations can be reduced to the set

$$
\begin{aligned}
& \alpha A\left(k^{2}-\beta^{2}\right)-\bar{\alpha} \bar{A}\left(k^{2}+\beta^{2}\right)=0, \\
& \mu A\left(4 k^{2} \alpha \beta-\left(k^{2}+\beta^{2}\right)^{2}\right) \\
& \quad-\bar{\lambda} \bar{A}\left(k^{2}-\bar{\alpha}^{2}\right)\left(k^{2}+\beta^{2}\right)=0 .
\end{aligned}
$$

For nontrivial solution on $A$ and $\bar{A}$, the determinant of coefficients $A$ and $\bar{A}$ in (31) and (32) must vanish,

$\left|\begin{array}{cc}\alpha\left(k^{2}-\beta^{2}\right) & -\bar{\alpha}\left(k^{2}+\beta^{2}\right) \\ \mu\left(4 k^{2} \alpha \beta-\left(k^{2}+\beta^{2}\right)^{2}\right) & -\bar{\lambda}\left(k^{2}-\bar{\alpha}^{2}\right)\left(k^{2}+\beta^{2}\right)\end{array}\right|=0$.

This leads to the relation of phase velocity $c$, i.e.,

$$
\begin{aligned}
1 & =\frac{\rho}{\bar{\rho}}\left(\frac{c_{\mathrm{T}}}{c}\right)^{4} \frac{\sqrt{1-\left(c / \bar{c}_{L}\right)^{2}}}{\sqrt{1-\left(c / c_{\mathrm{L}}\right)^{2}}} \\
& \times\left[4 \sqrt{\left(1-\frac{c^{2}}{c_{T}^{2}}\right)\left(1-\frac{c^{2}}{c_{\mathrm{L}}^{2}}\right)}-\left(2-\frac{c^{2}}{c_{T}^{2}}\right)^{2}\right] .
\end{aligned}
$$

Not surprisingly, this is exactly the nondispersive velocity equation of the Scholte waves in Cartesian coordinates [19-24]. It concludes that the Scholte waves have the same velocity with both cylindrical and Cartesian coordinate systems. However, the solutions used for displacements in the Bessel functions in the case of axisymmetric Scholte waves will be different from Cartesian coordinate solutions in trigonometric functions as shown in earlier studies. 
Naturally, the ultimate forms of the displacement field and stress field of Scholte waves with the cylindrical coordinate systems are

$$
\begin{aligned}
u_{r} & =k p A\left(\frac{2 q \mathrm{e}^{-2 \pi q z / \varsigma}}{1+q^{2}}-\frac{\mathrm{e}^{-2 \pi p z / \varsigma}}{p}\right) \\
& \times J_{1}\left(\frac{2 \pi r}{\varsigma}\right) \mathrm{e}^{\mathrm{i} \omega t}, \\
\bar{u}_{z} & =k p A \frac{1-q^{2}}{1+q^{2}} \mathrm{e}^{2 \pi \bar{p} z / \varsigma} J_{0}\left(\frac{2 \pi r}{\varsigma}\right) \mathrm{e}^{\mathrm{i} \omega t}, \\
u_{z} & =k p A\left(\frac{2}{1+q^{2}} \mathrm{e}^{-2 \pi q z / \varsigma}-\mathrm{e}^{-2 \pi p z / \varsigma}\right) \\
& \times J_{0}\left(\frac{2 \pi r}{\varsigma}\right) \mathrm{e}^{\mathrm{i} \omega t},
\end{aligned}
$$

and

$$
\begin{aligned}
\bar{\sigma}_{z} & =k^{2} \mu A\left(\left(1+q^{2}\right)-\frac{4 p q}{1+q^{2}}\right) \mathrm{e}^{2 \pi \bar{p} z / \varsigma} \\
& \times J_{0}\left(\frac{2 \pi r}{\varsigma}\right) \mathrm{e}^{\mathrm{i} \omega t} \\
\sigma_{z} & =k^{2} \mu A\left(\left(1+q^{2}\right) \mathrm{e}^{-2 \pi p z / \varsigma}-\frac{4 p q}{1+q^{2}} \mathrm{e}^{-2 \pi q z / \varsigma}\right) \\
& \times J_{0}\left(\frac{2 \pi r}{\varsigma}\right) \mathrm{e}^{\mathrm{i} \omega t}, \\
\tau_{z r} & =2 p k^{2} \mu A\left(\mathrm{e}^{-2 \pi p z / \varsigma}-\mathrm{e}^{-2 \pi q z / \varsigma}\right) \\
& \times J_{1}\left(\frac{2 \pi r}{\varsigma}\right) \mathrm{e}^{\mathrm{i} \omega t},
\end{aligned}
$$

where $\varsigma$ is the wavelength and $k \varsigma=2 \pi$.

The complete solutions of the Scholte waves in axisymmetric mode have been presented in this study. With the known properties of two materials, one can systematically calculate the phase velocity along with displacement and the stress fields. Undoubtedly, the solutions in the Bessel functions will reveal some special features of the Scholte waves in the structure.

\section{Numerical examples}

Now we consider the axisymmetric Scholte waves in a water-rock interface as an example. As in Fig. 1, Materials 1 and 2 are water and rock with properties [25-28]:

$$
\begin{array}{ll}
\bar{\rho}=998 \mathrm{~kg} / \mathrm{m}^{3}, & \bar{\lambda}=2.195 \times 10^{9} \mathrm{~Pa}, \\
\rho=2850 \mathrm{~kg} / \mathrm{m}^{3}, & \lambda=21.507 \times 10^{9} \mathrm{~Pa}, \\
\mu=21.506 \times 10^{9} \mathrm{~Pa}, &
\end{array}
$$

which yields

$$
\begin{aligned}
& \bar{c}_{\mathrm{L}}=1483 \mathrm{~m} / \mathrm{s}, \quad c_{\mathrm{L}}=4758 \mathrm{~m} / \mathrm{s}, \\
& c_{\mathrm{T}}=2747 \mathrm{~m} / \mathrm{s} .
\end{aligned}
$$

Then, the phase velocity of the Scholte waves is calculated from (34) as $c=1475.6349 \mathrm{~m} / \mathrm{s}$. Therefore, the parameters are $\bar{p}=0.0995, p=0.9507$, $q=0.8435$.

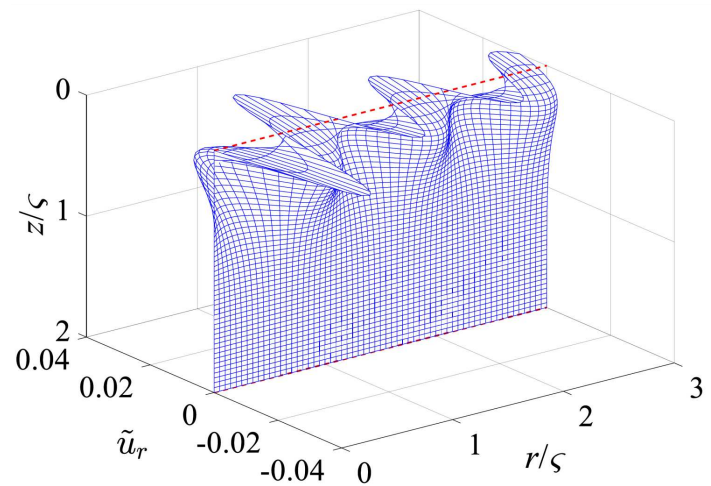

Fig. 2. The displacement $\widetilde{u}_{r}$ vs the normalized coordinate $r / \varsigma$ and $z / \varsigma$ at $t=0$.

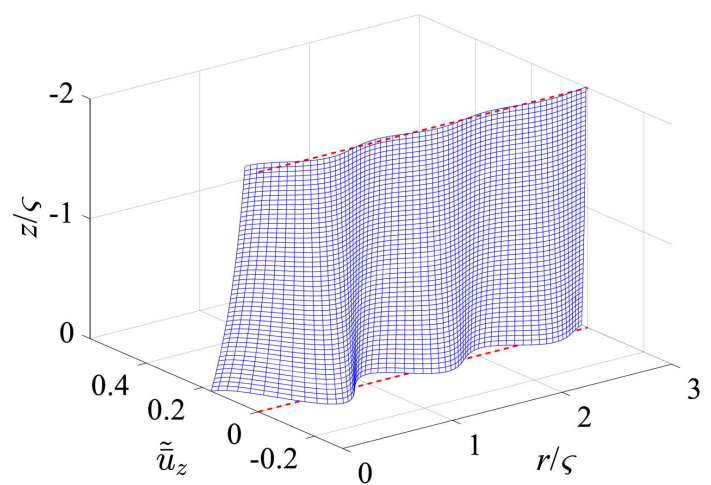

Fig. 3. The displacement $\widetilde{\widetilde{u}}_{z}$ vs the normalized coordinate $r / \varsigma$ and $z / \varsigma$ at $t=0$.

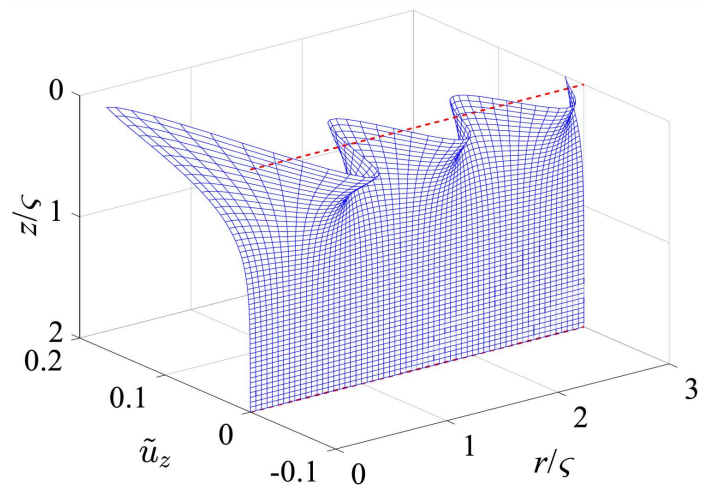

Fig. 4. The displacement $\widetilde{u}_{z}$ vs the normalized coordinate $r / \varsigma$ and $z / \varsigma$ at $t=0$.

For convenience in calculations, displacements (35)-(37) are normalized with $k p A$. Respectively, these are denoted as $\widetilde{u}_{r}=u_{r} /(k p A)$, $\widetilde{\bar{u}}_{z}=\bar{u}_{z} /(k p A), \widetilde{u}_{z}=u_{z} /(k p A)$. In turn, stresses (38)-(40) are normalized with $k^{2} \mu A$ and are denoted as $\widetilde{\bar{\sigma}}_{z}=\bar{\sigma}_{z} /\left(k^{2} \mu A\right), \quad \widetilde{\sigma}_{z}=\sigma_{z} /\left(k^{2} \mu A\right)$, $\widetilde{\tau}_{z r}=\tau_{z r} /\left(k^{2} \mu A\right)$.

Then, the normalized displacements for the Scholte waves of the structure in Fig. 1 at $t=0$ are plotted in Figs. 2-4. 


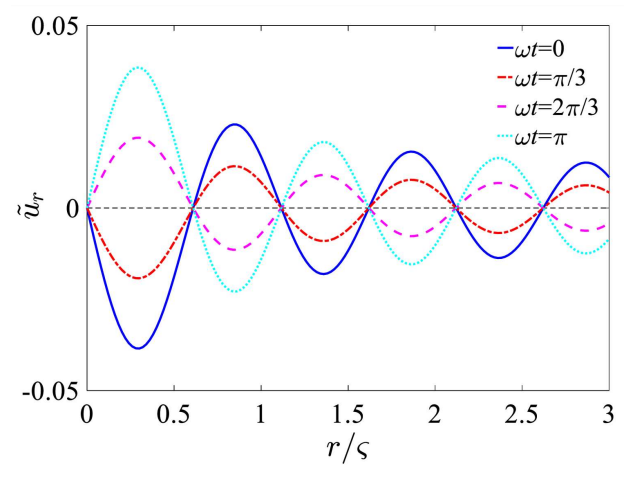

Fig. 5. The normalized displacement $\widetilde{u}_{r}$ vs $r / \varsigma$ at $z=0$.

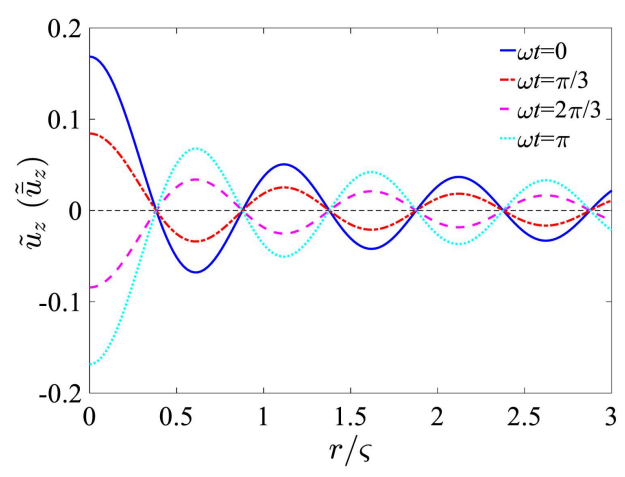

Fig. 6. The normalized displacement $\widetilde{u}_{z}\left(\right.$ or $\left.\bar{u}_{z}\right)$ vs $r / \varsigma$ at $z=0$.

The normalized displacements at $z=0$ are plotted in Figs. 5 and 6.

With $r=1.25 \varsigma$, the displacement $\widetilde{u}_{r}$ varied in time with the depth as depicted in Fig. 7. The nodal point $G$ has the value $y_{g}=0.0965$. The maximum displacements below the interface are marked as points $F_{1}, F_{2}, F_{3}$ and $F_{4}$ with $y_{f}=0.2747$ for different time. The maximum displacements in the interface are also marked as $E_{1}, E_{2}, E_{3}$ and $E_{4}$.

Simultaneously, the displacements $\widetilde{\bar{u}}_{z}$ and $\widetilde{u}_{z}$ are depicted in Fig. 8. However, there is no nodal point of displacement in the $z$-direction. The maximum displacements at the interface are marked as $M_{1}$, $M_{2}, M_{3}$ and $M_{4}$ with positions $x_{m 1}=2 x_{m 2}=$ $-2 x_{m 3}=-x_{m 4}=0.0344$.

Finally, displacement solutions (35)-(37) can be used for the discussion of axisymmetric Scholte waves in the far field. From a strictly mathematical point of view, the wave velocity is defined only by the function $f[k(r-c t)]$. Clearly it can be seen at this stage that displacements $\widetilde{u}_{r}, \widetilde{\bar{u}}_{z}$ and $\widetilde{u}_{z}$ do not have this form. As it is known, however, the Bessel functions can be written in the following asymptotic form:

$$
\begin{aligned}
& J_{1}(x)=\sqrt{\frac{2}{\pi x}} \sin \left(x-\frac{\pi}{4}\right), \\
& J_{0}(x)=\sqrt{\frac{2}{\pi x}} \cos \left(x-\frac{\pi}{4}\right) .
\end{aligned}
$$

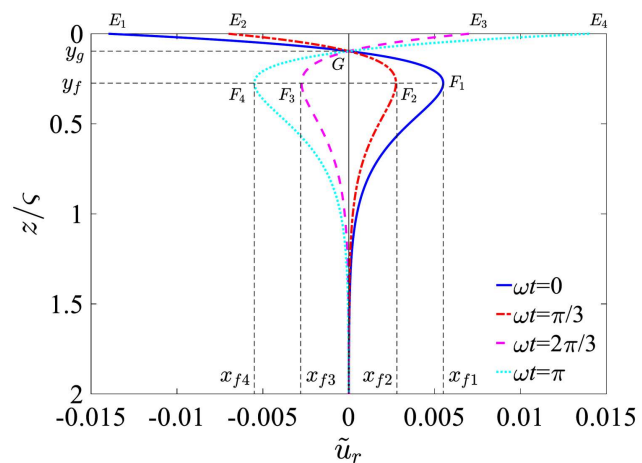

Fig. 7. The displacement $\widetilde{u}_{r}$ at $r / \varsigma=1.25$ in the depth direction.

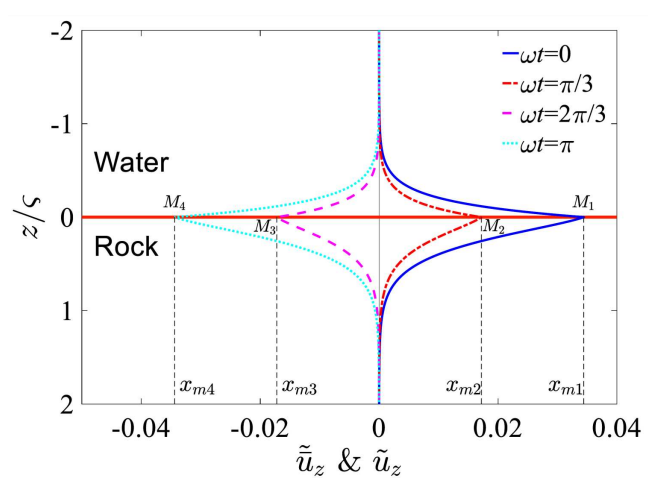

Fig. 8. The displacement $\widetilde{\bar{u}}_{z}$ and $\widetilde{u}_{z}$ at $r / \varsigma=1.25$ in the depth direction.

With this approximation, and for the large radius $r,(35)-(37)$ can be written as

$$
\begin{aligned}
& u_{r}=a(z) \sin (k r-\pi / 4) \cos (\omega t), \\
& \bar{u}_{z}=\bar{b}(z) \cos (k r-\pi / 4) \cos (\omega t), \\
& u_{z}=b(z) \cos (k r-\pi / 4) \cos (\omega t),
\end{aligned}
$$
with

$$
\begin{aligned}
& a(z)=p A \sqrt{\frac{2 k}{\pi r}}\left(\frac{2 q}{1+q^{2}} \mathrm{e}^{-\beta z}-\frac{1}{p} \mathrm{e}^{-\alpha z}\right) \\
& \bar{b}(z)=p A \sqrt{\frac{2 k}{\pi r}} \frac{1-q^{2}}{1+q^{2}} \mathrm{e}^{\bar{\alpha} z} \\
& b(z)=p A \sqrt{\frac{2 k}{\pi r}}\left(\frac{2}{1+q^{2}} \mathrm{e}^{-\beta z}-\mathrm{e}^{-\alpha z}\right) .
\end{aligned}
$$

Applying the basic identities of trigonometric functions, (43)-(45) can be rewritten as

$$
\begin{aligned}
u_{r} & =\frac{a(z)}{2}[\sin (k r-\pi / 4+\omega t) \\
& +\sin (k r-\pi / 4-\omega t)], \\
\bar{u}_{z} & =\frac{\bar{b}(z)}{2}[\cos (k r-\pi / 4+\omega t) \\
& +\cos (k r-\pi / 4-\omega t)],
\end{aligned}
$$




$$
\begin{gathered}
u_{z}=\frac{b(z)}{2}[\cos (k r-\pi / 4+\omega t) \\
+\cos (k r-\pi / 4-\omega t)],
\end{gathered}
$$

which are in the waveform of $f[k(r-c t)]$ and are the superposition of one forward traveling wave with one backward traveling wave with the wave velocity $c=\omega / k$. Therefore, the axisymmetric Scholte waves exhibit the property of traveling waves in the far field away from the origin. Furthermore, the displacements in the far field display a $1 / \sqrt{r}$ decaying, while it is invariant in Cartesian coordinates. Actually it can be explained by the density of the energy. As the axisymmetric Scholte waves travel from the source, the energy is scattered around the cylindrical surface in which each infinitesimal area possesses the energy proportional to1/ $(2 \pi r)$ if there is no energy loss in the propagation. Since the displacements are directly proportional to the square root of energy, the displacements are directly proportional to $1 / \sqrt{r}$. For a large radius, the axisymmetric Scholte waves can be regarded as planar surface waves in a small range.

With $z=0$, using the displacement $\widetilde{u}_{r}$ plots in Fig. 9, and mark point $N$ as the first intersection between the exact and asymptotic curves, it is found that $x_{n}=0.3930$ and $y_{n}=-0.0334$. Using the numerical comparisons in Table I, the accuracy of different solutions is clearly shown. Similar comparisons can also be made for other displacement solutions.

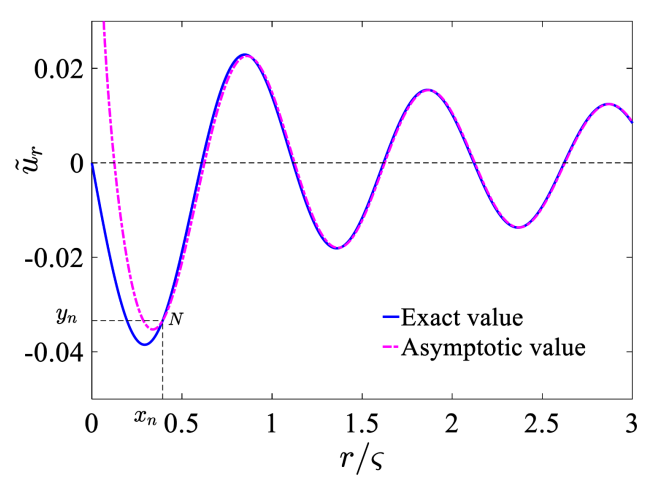

Fig. 9. A comparison of $\widetilde{u}_{r}$ with exact and asymptotic values at $z=0$ and $t=0$.

TABLE I

A comparison of $\widetilde{u}_{r}$ with exact and asymptotic values at $z=0$ and $t=0$

\begin{tabular}{c|c|c|c}
\hline \hline$r / \varsigma$ & Exact value & Asymptotic value & Error [\%] \\
\hline 0.5 & -0.0188 & -0.0211 & 11.84 \\
1 & 0.0141 & 0.0149 & 5.98 \\
1.5 & -0.0117 & -0.0122 & 3.99 \\
2 & 0.0102 & 0.0105 & 2.99 \\
2.5 & -0.0092 & -0.0094 & 2.39 \\
3 & 0.0084 & 0.0086 & 1.99
\end{tabular}

The results above indicated that the axisymmetric Scholte waves can be plane waves in the far field, consistent with the results from Cartesian coordinates. Besides, it also illustrates that the displacement near the origin or the excitation point is larger than the outer region which is represented by the solution in Cartesian coordinates. To study waves generated by point sources, evidently axisymmetric coordinates and solutions must be used for a better understanding of the wave propagation, especially near the wave source, or in near field.

\section{Conclusions}

With systematically deduced solutions of the Scholte waves in cylindrical coordinates it is concluded that the wave velocity still maintains the usual value as with Cartesian coordinates. As expected, with properties of the Bessel functions, displacements in the two half-spaces are attenuated in the new form in contrast to the constant values known in Cartesian coordinates. In other words, the trajectory of particle polarization of the Scholte waves near the origin is distinct from Cartesian coordinates, but the asymptotic expansion of the Bessel function ensures that the displacement amplitude will remain the same as Cartesian coordinates when the radius is large or far from the origin. The results further show that the axisymmetric solution near the origin is larger than the solution given by the trigonometric functions, implying that the axisymmetric description is more accurate in solving such problems in the vicinity of a point source. In the case of a wavelength of kilometers, a better solution with a radius of kilometers should be obtained by using the cylindrical coordinate formula proposed in this study.

Moreover, these results of the Bessel functions could also serve as the basis for the study of fluid sensors and actuators of finite structures subjected to the Scholte waves, such as in seabed explorations. The Scholte waves tested and measured from a point source also required a better understanding on the displacement changes near and far from the origin for the estimation of damping. The solutions and analysis in this study are undoubtedly a good start for studying the Scholte waves in curved structures, especially in circular devices, where a systematic optimization is always expected.

\section{Acknowledgments}

This research was partially supported by the National Key Research and Development Program (Grant No. 2017YFB1102900), the Ministry of Science and Technology of China and the National Natural Science Foundation of China (Grant No. 11672142). 
The additional support was through the Technology Innovation 2025 Program (Grant No. 2019B10122) of the Municipality of Ningbo, the Research and Development Program of Key Disciplines of Guangdong Province (Grant No. 2020B0101040002), and the Research and Development Program in Key Disciplines of Hunan Province (Grant No. 2019GK2111).

\section{References}

[1] J.G. Scholte, Proc. Konink. Nederl. Akad. Weten Schappen 45, 159 (1942).

[2] R. Stoneley, Proc. R. Soc. Lond. A 106, 416 (1924).

[3] L. Cagniard, Reflection and Refraction of Progressive Seismic Waves, McGraw-Hill, 1962.

[4] W.M. Ewing, W.S. Jardetzky, F. Press, Elastic Waves in Layered Media, Mc GrawHill, 1957.

[5] J.D. Achenbach, Wave Propagation in Elastic Solids, North-Holland, 1973.

[6] K.F. Graff, Wave Motion in Elastic Solids, Dover, 1975.

[7] A.C. Eringen, Elastodynamics, Vol. 2, Linear Theory, Academic Press, 1975.

[8] J.L. Rose, Ultrasonic Guided Waves in Solid Media, Cambridge University Press, 2014.

[9] J. Wang, J.B. Lin, J. Intell. Mater. Syst. Struct. 16, 623 (2005).

[10] J. Wang, J.B. Lin, Y.P. Wan, Z, Zhong, Int. J. Appl. Electromagn. Mech. 22, 53 (2005).

[11] J. Wang, K.Y. Hashimoto, J. Sound Vibrat. 295, 838 (2006).

[12] J. Wang, J.K. Du, Q.Q. Pan, Sci. China Phys. Mech. 10, 631 (2007).
[13] J. Wang, S.Y. Wang, L.T. Xie, Y.Y. Zhang, L.L. Yuan, J.K. Du, H. Zhang, Theor. Appl. Mech. Lett. 10, 120 (2020).

[14] C.L. Bian, B. Huang, L.T. Xie, L.J. Yi, L.L. Yuan, J. Wang, Acta Phys. Pol. A 139, 124 (2021).

[15] K. Sezawa, K. Kanai, Bull. Earthquake Res. Inst. Tokyo Univ. 17, 1 (1939).

[16] J.G. Scholte, Geophys. J. In. 5, 120 (1947).

[17] P. Sofer, M. Sofer, J. Gebauer, P. Niegodajew, K. Gruszka, Acta Phys. Pol. A 138 , 272 (2020).

[18] D. Mitchell, J.T. Gau, Eur. J. Mech. A Solids 75, 216 (2019).

[19] R.M.A. Stoneley, Geophys. Suppl. Monthly Notices R. Astron. Soc. 1, 527 (1928).

[20] A.S. Ginzbarg, E. Strick, Bull. Seismol. Soc. Am. 48, 51 (1958).

[21] M. Pasternak, Acta Phys. Pol. A 116, 371 (2009).

[22] P. Chadwick, J. Elast. 6, 73 (1976).

[23] D. Royer, E. Dieulesaint, Elastic Waves in Solids I and II, Springer, 2000.

[24] J. Kaplunov, D. Prikazchikov, Adv. Appl. Mech. 50, 1 (2017).

[25] E. Flores-Mendez, M. Carbajal-Romero, N. Flores-Guzmán, R. Sánchez-Martínez, A. Rodríguez-Castellanos, J. Appl. Math. 313207, 1 (2012).

[26] J. Qian, C.P. Li, L.R. Qian, M.J. Li, H.J. Li, B.H. Yang, Diam. Relat. Mater. 88, 123 (2018).

[27] M.T. Chen, Q. Huan, F.X. Li, J. Acoust. Soc. Am. 146, 482 (2019).

[28] G. Dal Moro, Sci. Rep. (UK) 10, 211 (2020). 